\section{Case Reports in Ophthalmology}

\title{
The Limitation of Applying Heat to the External Lid Surface: A Case of Recalcitrant Meibomian Gland Dysfunction
}

\author{
Christen J. Kenrick Sabiha S. Alloo
}

Korb Research, Boston, MA, USA

\section{Keywords}

Dry eye $\cdot$ Meibomian gland dysfunction $\cdot$ Bruder mask $\cdot$ Blephasteam $\cdot$ MiBoFlo $\cdot$ LipiFlow

\begin{abstract}
The effects on the inner surface temperatures of the upper and lower eyelids of four commercial heat therapies were compared for an individual with recalcitrant meibomian gland dysfunction. Three therapies (Bruder mask, Blephasteam, and MiBoFlo) involved the application of heat to the external lid surface, and the fourth (LipiFlow) applied heat to the internal lid surface. Only LipiFlow was effective in elevating the inner surface temperatures to the reported $40^{\circ} \mathrm{C}$ therapeutic threshold for melting obstructed meibum.

(C) 2017 The Author(s)

Published by S. Karger AG, Basel
\end{abstract}

\section{Introduction}

Dry eye symptoms are one of the leading reasons why patients seek eye care in the United States [1]. The prevalence of aqueous-deficient disease is far exceeded by that of evaporative dry eye, the primary cause of which is meibomian gland dysfunction (MGD), with MGD present in $86 \%$ of all dry eye patients of known cause [2, 3]. First-line treatment of MGD usually includes some form of heat therapy, attempting to alleviate terminal duct 


\section{Case Reports in Ophthalmology}

obstruction by liquefaction of the gland contents [4]. However, this therapy can be challenging for many reasons.

A primary challenge is that MGD alters the molecular composition of the meibum increasing the melting point relative to the normal body temperature [5]. This increase results in the need for higher temperatures within the gland to liquefy obstructive material. Previous in vitro and in vivo studies have demonstrated that although the temperatures required to melt the obstructive secretions are reported to range from 32 to $45^{\circ} \mathrm{C}$, the more severely obstructed glands present in MGD require temperatures $>40^{\circ} \mathrm{C}$ for effective liquefaction [5]. Achieving this temperature within the meibomian glands presents several concerns. One obstacle is that there is an approximate $5^{\circ} \mathrm{C}$ difference in temperature between heat applied on the external eyelid surfaces and that which reaches the inner surface of the lids (palpebral conjunctiva), where the meibomian glands are located [6]. This difference is due to both dissipation of heat while passing through the lid tissues and to constant movement of blood through vasculature wicking heat away from the lids [7]. Therefore, achieving the desired temperature of $40^{\circ} \mathrm{C}$ at the palpebral conjunctiva requires a constant heat of at least $45^{\circ} \mathrm{C}$ be maintained on the outer lid surface, a temperature which may be both uncomfortable and risk causing thermal injury to the eyelid skin [8]. A further conundrum is that while adequate heat is required to liquefy obstructions, the cornea is not designed to withstand temperatures much above body temperature. When subjected to heat $>40^{\circ} \mathrm{C}$, the corneal tissue becomes more malleable, and therefore more vulnerable to molding when exposed to any external pressure [9]. Further, the nature of the obstructive material, particularly when of longstanding, may require a higher temperature than $40^{\circ} \mathrm{C}$ at the palpebral conjunctiva [5].

Herein, we describe a case of recalcitrant and symptomatic MGD, where lid warming was deemed necessary but had previously been ineffective, which prompted evaluation and comparison of four different commercially available heat therapies in regard to their effects on the inner surface temperatures of the eyelids.

\section{Case Report}

A 28-year-old woman, an optometrist, was self-referred for the evaluation of bilateral eye irritation. She reported symptoms characteristic of dry eye: stinging, foreign body sensation, and tearing. These symptoms were present with and without contact lens wear. She also reported a decrease in the overall comfort of her contact lenses and decreased contact lens wear time. She was particularly concerned by a nightly burning sensation upon removal of her contact lenses. Her score on the Standard Patient Evaluation of Eye Dryness (SPEED) questionnaire was 11 points of a possible 28 points. She noted that her symptoms first occurred between 1 and 2 years prior to her visit and were increasing in severity. She had previously trialed warm compresses at home without success and was fearful that her dry eye symptoms would worsen and that she would lose the ability to continue wearing contact lenses. She reported no history of systemic disease and denied any current medications.

On initial presentation, her eyes appeared white and quiet. The eyelashes were normal without madarosis, blepharitis, seborrheic scurf, or collarettes. Fluorescein tear break-up time was $4 \mathrm{~s}$ for the right eye and $6 \mathrm{~s}$ for the left as measured by the Dry Eye Test method [10]. There was no corneal staining and no significant conjunctival staining detected following the instillation of two drops of sodium fluorescein, the second drop instilled 5 min after the first, followed by evaluation 1 min later. Several meibum plugs, minimally protruding and obstructing the gland orifices, were observed on each lower lid. The total number of 


\section{Case Reports in Ophthalmology}

meibomian glands yielding liquid secretion, as evaluated with the Meibomian Gland Evaluator (TearScience, Morrisville, NC, USA), was 4 for the right lower lid and 10 for the left lower lid. No significant structural changes were observed by meibography. The primary etiology of the patient's symptoms was diagnosed as MGD.

Prior studies have shown that the application of external heat has limited benefits, and that not all methods are equally efficacious [11]. We therefore questioned whether home therapies in general could achieve the temperature elevation necessary to provide benefit to this patient. As the patient was herself an optometrist and very interested in an evidencebased treatment plan, we elected to compare two commercial methods of home heat therapy for their ability to elevate the temperature at the meibomian glands under carefully controlled conditions, where the authors were present and personally attentive to all procedures. The two home therapies compared were the Bruder mask (Bruder Healthcare, Alpharetta, GA, USA) and Blephasteam (Théa, Clermont-Ferrand, France). Additionally, we elected to compare two office procedures for MGD, one with an externally applied heat source: MiBoFlo (Mibo Medical, Dallas, TX, USA), and the second with heat applied to the inner surface of the eyelid: LipiFlow (TearScience, Morrisville, NC, USA).

The purpose of this testing was to evaluate the efficacy of the heat transfer from the device to the meibomian glands. As the meibomian glands are located adjacent to the inner surface of the eyelid, the temperature of the palpebral conjunctiva served as the primary endpoint in determination of which heat therapy offered the best possibility of successful treatment. Temperature was measured immediately prior to and immediately after application of each therapy using a non-contact infrared thermometer (Innoo Tech, Shenzhen, China). The temperature of the palpebral conjunctiva was measured by manually everting the eyelid and then taking a reading as quickly as possible (within 2-3 s) to minimize heat loss. To facilitate measurements, only the right upper and lower eyelids were measured. Each therapy was assessed at a different visit, with visits separated by a minimum of $4 \mathrm{~h}$, to ensure that temperatures would have returned to basal levels and would not be influenced by prior treatments. The devices were tested in order of increasing complexity: Bruder mask, Blephasteam, MiBoFlo, LipiFlow. All therapies were performed following each manufacturer's instructions precisely.

At the first visit, the Bruder mask was evaluated. The mask was heated in a microwave for $20 \mathrm{~s}$ and applied to the outer surface of the eyelids for a period of $10 \mathrm{~min}$. Prior to application, the surface temperature of the mask was variable, ranging from 38 to $48^{\circ} \mathrm{C}$, with the warmest areas closest to the center of the mask and the coolest at the outer edges. The patient's eyes remained closed throughout the treatment. At the conclusion of the treatment period, palpebral conjunctival temperatures had increased from 36.7 to $38.3^{\circ} \mathrm{C}$ and from 36.2 to $37.1^{\circ} \mathrm{C}$ for the lower and upper eyelids, respectively. These results are consistent with previously published findings [11].

At the second visit, the Blephasteam was evaluated. The device was allowed to preheat until a flashing light indicated treatment could begin. The temperature of the Blephasteam itself is not known, but a prior report indicates that the temperature of the outer lid after the application can be as high as $42^{\circ} \mathrm{C}$ [12]. Two inserts were moistened with saline solution and placed inside the mask prior to commencing the 10-min treatment. The patient's eyes were open within the mask, allowing uninhibited blinking throughout the treatment. At the conclusion of the treatment period, palpebral conjunctival temperatures had increased from 37.0 to $39.2^{\circ} \mathrm{C}$ and from 36.5 to $37.6^{\circ} \mathrm{C}$ for the lower and upper eyelids, respectively. These results are consistent with previously published findings $[11,12]$. 


\section{Case Reports in Ophthalmology}

At the third visit, the MiBoFlo was evaluated. MiBoFlo uses an external paddle that is heated to a temperature of $108^{\circ} \mathrm{F}\left(42.2^{\circ} \mathrm{C}\right)$. The device was allowed to preheat until the display indicated that treatment could begin. A small amount of ultrasound gel was applied to the instrument's heated tip prior to gently massaging the outer skin of the upper and lower eyelids for a period of $12 \mathrm{~min}$, as per the recommendation of the manufacturer. The patient's eyes remained closed throughout the treatment period. At the conclusion of the treatment period, the palpebral conjunctival temperature of the lower eyelid was unchanged compared to pre-treatment measurement, remaining stable at $36.8^{\circ} \mathrm{C}$. The palpebral conjunctival temperature of the upper eyelid increased very minimally from 36.3 to $36.5^{\circ} \mathrm{C}$. Upon review of the literature, no prior publications could be found describing the effects of MiBoFlo on the temperature of the palpebral conjunctiva, and thus this case appears to be the first providing comparative data relating to the efficacy of heat transfer from the device to the inner surface of the eyelid.

At the fourth visit, the LipiFlow was evaluated. LipiFlow is fundamentally different from the other heat therapies in that it combines heat with directional pressure $[13,14]$. The effects of this combined technology were not evaluated in this case, but rather only the efficacy of heat transfer to the palpebral conjunctiva. The activators were inserted into each eye and the treatment program was initiated. The patient's eyes remained closed throughout the standard 12-min treatment. At the conclusion of the treatment period, palpebral conjunctival temperatures had increased from 37.0 to $42.0^{\circ} \mathrm{C}$ and from 36.9 to $41.1^{\circ} \mathrm{C}$ for the lower and upper eyelids, respectively. These results are consistent with previously published findings [13].

The average changes in palpebral conjunctival temperatures for each treatment option are represented in Figure 1. LipiFlow was the only treatment that increased the temperature of the palpebral conjunctiva above $40^{\circ} \mathrm{C}$. Since home therapies did not achieve this temperature, and whereas home treatment had not been effective in the past, the patient elected not to use any home therapy, although she was informed that there could be some additional benefit. At the progress evaluation 3 months later, the patient reported improved comfort with and without contact lenses, decreased foreign body sensation, decreased tearing, and a resolution of the burning sensation she had felt upon nightly removal of contact lenses. Her score on the SPEED questionnaire at this visit was 4 points of a possible 28 points, an improvement compared to her score of 11 points at her initial evaluation.

\section{Discussion}

Given that the anatomic position of the meibomian glands is adjacent to the palpebral conjunctiva, and therefore closer to the inner eyelid surface than the outer surface, it is reasonable to expect that application of heat to the inner eyelid surface would be more effective in increasing the temperature of the glands [15]. Thus, it is not surprising that LipiFlow was the only therapy which elevated the temperature above the target value of $40^{\circ} \mathrm{C}$. The potential problem of heating the inner eyelid surface without raising the temperature of the cornea is achieved by the LipiFlow activators in a unidirectional heating surface that simultaneously insulates the ocular surface $[13,14]$.

As illustrated by this case, attempting to heat the inner surface of the eyelids with a heat source applied to the external lid surface has limited efficacy. There may be palliative effects seen from any of these forms of external lid surface heating, but these are likely secondary to factors other than liquefaction of the meibum and amelioration of the obstruction within the 
gland. While there is evidence to support that Bruder mask and Blephasteam are capable of elevating the temperature of the palpebral conjunctiva, there is no evidence that the MiBoFlo is capable of doing so $[11,12]$.

There may be some benefit from the use of the Bruder mask or Blephasteam, as both of these did raise the palpebral conjunctival temperature in this patient, although not to the desired therapeutic level of $40^{\circ} \mathrm{C}[11,12]$. These therapies should be considered as supplemental home therapy, which can be used daily for 10-15 min, as supplements to office treatment to remove meibomian gland obstructions.

An inherent limitation of all case reports is the evaluation of a single individual, but in this instance the patient's interest and willingness to participate in these temperature comparisons has provided an unusually valuable opportunity. A limitation was that measurements of the palpebral conjunctiva required eversion of the target eyelid, which increases evaporation and thereby decreases temperature. Ideally, the temperature of both the inner and outer eyelid surfaces would be measured continuously, so as to provide a more comprehensive evaluation of the heat transfer for each therapy. Multiple studies have evaluated the efficacy of using heat alone to treat MGD [4]. The safety and efficacy of LipiFlow has also been studied extensively $[13,14]$. This case report validates these studies, but is novel in its same-subject comparison and inclusion and evaluation of MiBoFlo. It is our hope that this case report and data will stimulate further studies.

\section{Statement of Ethics}

Written consent was obtained from the patient for publication of this case report.

\section{Disclosure Statement}

The authors have no conflicts of interest to report regarding this case report.

\section{References}

1 Schaumberg DA, Sullivan DA, Buring JE, Dana MR: Prevalence of dry eye syndrome among US women. Am J Ophth 2003;136:318-326.

-2 Lemp MA, Crews LA, Bron AJ, Foulks GN, Sullivan BD: Distribution of aqueous-deficient and evaporative dry eye in a clinic-based patient cohort: a retrospective study. Cornea 2012;31:472-478.

-3 Nichols KK, Foulks GN, Bron AJ, et al: The international workshop on meibomian gland dysfunction: executive summary. Invest Ophth and Vis Sci 2011;52:1922-1929.

-4 Geerling G, Tauber J, Baudouin C, et al: The international workshop on meibomian gland dysfunction: report of the subcommittee on management and treatment of meibomian gland dysfunction. Invest Ophth and Vis Sci 2011;52:2050-2064.

-5 Borchman D, Foulks GN, Yappert MC, et al: Human meibum lipid conformation and thermodynamic changes with meibomian-gland dysfunction. Invest Ophthalmol Vis Sci 2011;52:3805-3817.

6 Blackie CA, Solomon JD, Greiner JV, Holmes M, Korb DR: Inner eyelid surface temperature as a function of warm compress methodology. Optom Vis Sci 2008;85:675-683.

7 Despa F, Orgill DP, Neuwalder J, Lee RC: The relative thermal stability of tissue macromolecules and cellular structure in burn injury. Burns 2005;31:568-577.

8 Moritz AR, Henriques FC: Studies of thermal injury: the relative importance of time and surface temperature in the causation of cutaneous burns. Am J Path 1947;23:695-720.

-9 McMonnies CW, Korb DR, Blackie CA: The role of heat in rubbing and massage-related corneal deformation. Cont Lens Anterior Eye 2012;35:148-154. 
Kenrick and Alloo: The Limitation of Applying Heat to the External Lid Surface: A Case of Recalcitrant Meibomian Gland Dysfunction

10 Korb DR, Greiner JV, Herman J: Comparison of fluorescein break-up time measurement reproducibility using standard fluorescein strips versus the Dry Eye Test (DET) method. Cornea 2001;20:811-815.

11 Murakami DK, Blackie CA, Korb DR: All warm compresses are not equally efficacious. Optom Vis Sci 2015;92:e327-e333.

12 Pult H, Riede-Pult BH, Purslow C: A comparison of an eyelid-warming device to traditional compress therapy. Optom Vis Sci 2012;89:1-7.

13 Korb DR, Grenon SM, Willis TR, Gravely BT: TearScience Inc., assignee. Inner eyelid treatment for treating meibomian gland dysfunction. United States patent US 7981146 B2. 2011 Jul 19.

14 Blackie CA, Coleman CA, Holland EJ: The sustained effect (12 months) of a single-dose vectored thermal pulsation procedure for meibomian gland dysfunction and evaporative dry eye. Clin Ophthalmol 2016;10:1385-1396.

15 Knop E, Knop N, Millar T, Obata H, Sullivan DA: The international workshop on meibomian gland dysfunction: report of the subcommittee on anatomy, physiology, and pathophysiology of the meibomian gland. Invest Ophthalmol Vis Sci 2011;52:1938-1978.

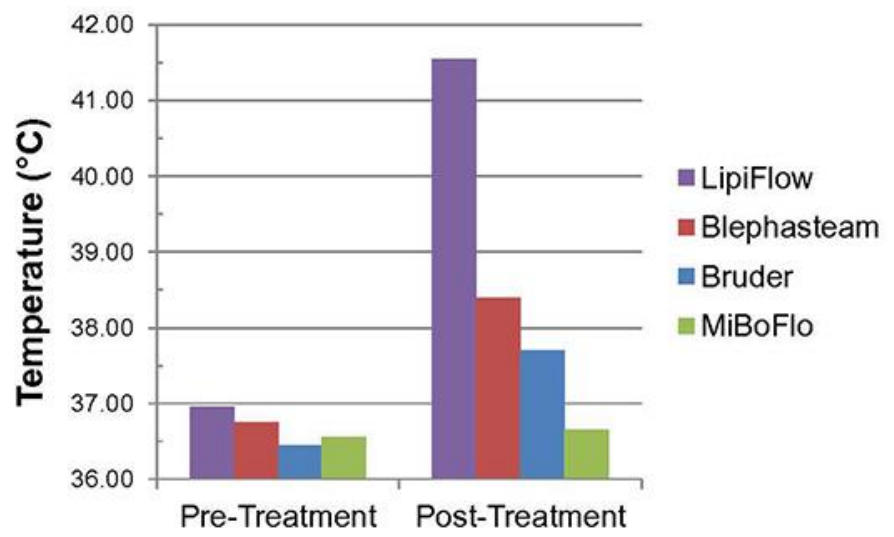

Fig. 1. Average palpebral conjunctival temperature of the upper and lower eyelids. 